Research Article

\title{
Torque Measurement of High-Precision Reducer for Industrial Robot
}

\author{
Zhen Yu $\mathbb{D}^{1}$ and Yuan Zhang $\mathbb{D}^{2}$ \\ ${ }^{1}$ State Key Laboratory of Precision Measuring Technology and Instrument, Tianjin University, China \\ ${ }^{2}$ School of Management, China University of Mining \& Technology (Beijing), China \\ Correspondence should be addressed to Yuan Zhang; solseagull@tju.edu.cn
}

Received 24 November 2020; Accepted 26 June 2021; Published 10 July 2021

Academic Editor: Pawel Malinowski

Copyright (c) 2021 Zhen Yu and Yuan Zhang. This is an open access article distributed under the Creative Commons Attribution License, which permits unrestricted use, distribution, and reproduction in any medium, provided the original work is properly cited.

\begin{abstract}
Torque testing is crucial to improve the quality of high-precision reducers-the core component of industrial robots. Herein, a torque-measurement system for a novel vertical measuring instrument is designed. The distance from the torque transducers to the robot reducer is minimized to ensure the shortest measurement chain. The symmetrical system structure improves the overall rigidity, and error compensation can be performed easily. The characteristics of the torque measurement errors due to shaft bending and torsional deformations were also analyzed. A torque calibrator comprising two high-precision torque output systems was used to calibrate torque transducers in the measurement system. Reasonable and practical compensation models based on a backpropagation neural network were developed to accurately obtain the input and output torques of the reducer. As the torque-measurement precision of the reducer detector reached $0.1 \%$ over the entire torque range, the instrument can be used for accuracy measurement of the input and output torques of the robot reducer.
\end{abstract}

\section{Introduction}

At present, industrial robots are widely applied in many areas, and the performance requirements of robot reducers are becoming increasingly more complex [1]. Therefore, the comprehensive performance detection of precision reducers has an essential impact on the development of robots [2]. Many experts and scholars have done much work on the internal parts of reducers [3] and the manufacturing errors of reducers $[4,5]$. Many experts and scholars have made some progress with the performance detection of precision reducers $[6,7]$. Performance parameters of reducers generally include torsional rigidity, transmission error, backlash, lost motion, and transmission efficiency [8-10]. The primary variable in the definitions of these main parameters includes torque, which is time-dependent. Therefore, it is necessary to measure torques of input and output shafts of the tested reducer in real time $[11,12]$.

Torque transducers are included in measurement modules to measure the input and output torques of the reducer. The drive and load systems are selected to be parts of the instrument according to testing functions. In terms of layout, the horizontal type is mainstream design. Sensors, driving devices, and other connecting components supported by cantilevers are fixed on guide rails or a horizontal base in series connection [13-17]. Figure 1 shows one of the commonly used layouts for the detector of robot reducers. It can be used for long-time monitoring of multiparameters.

For the instrument mentioned above, components must be installed on the guide rail to facilitate the installation and disassembly of multitype reducers. This design scheme seriously damages the overall torsional stiffness of the instrument. Furthermore, additional torques caused by bending and torsional deformation of the shafts are included in the measurement results. The uneven frictional force caused by deformation of the components and the misalignment error can lead to torque fluctuation. The above troubles inevitably disturb the measurements of torque [18-20].

The contribution of this paper is to introduce a torque measurement method based on the backpropagation (BP) neural network in the vertical precision robot reducer detector. Our work overcomes some shortcomings of existing schemes and achieves high-precision torque measurement in the full range. 


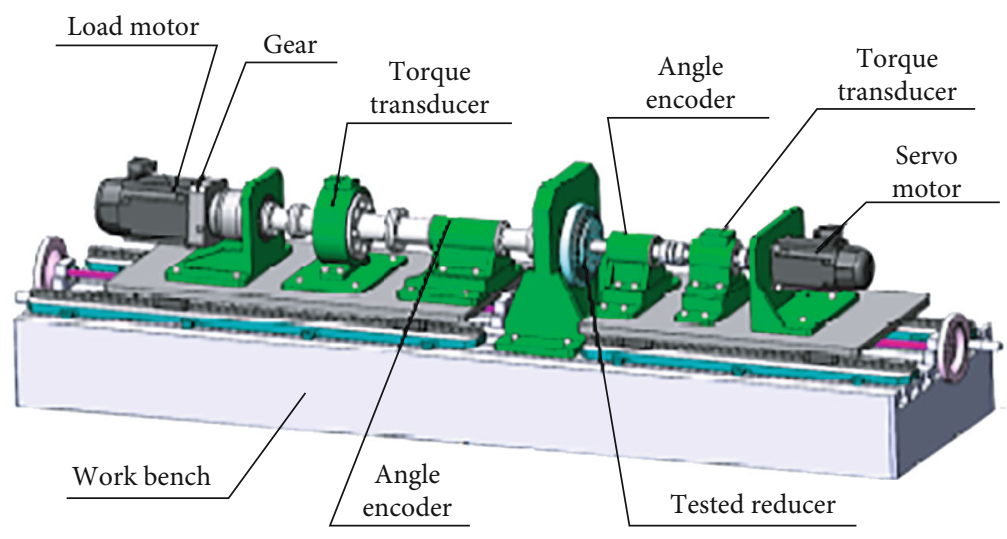

FIGURE 1: One of the commonly used layouts of instruments for reducer testing.

\section{Overview of Torque Measurement System}

In order to measure the input and output torques of the reducer, the torque measurement systems on input and output sides are designed, based on the novel vertical precision robot reducer detector, as shown in Figure 2. The instrument consists of two torque measurement modules: the Torque Measurement Module at the Input Side of the Reducer (TMMISR) and the Torque Measurement Module at the Output Side of the Reducer (TMMOSR). Each module comprises one motor, one torque transducer, one test mode conversion part, and some connecting parts. The red part in Figure 2 is the Tested Assemblies (TA). The robot reducer is installed inside the TA. The TA is used to achieve the quick installation of different types of reducers. The connections with the vertical precision robot reducer detector adopt a uniform and standard structure.

Torque transducers are closed to the measured target as much as possible to ensure the shortest measurement chain and least number of error sources. Both torque measurement systems use torque transducer of strain-resistor to obtain input and output torques of the robot reducer and convert frequency signals into torque through an algorithm. Each rotor in the torque transducer is installed with a shaft that is supported by a bearing. The misalignment error between the tested reducer and the rotor of the torque transducer should be less than $0.03 \mathrm{~mm}$.

When the torque is transmitted, the circumferential direction of the hollow shafts withstands the torque together. This method avoids stress concentration and misalignment error theoretically. Additionally, the torsion rigidity of a circle with an equal cross-section area per unit length is better than that of others. Hence, cylindrical and disc supports can significantly improve the structural stiffness and ensure measurement precision. Simultaneously, the plane assumption in the mechanics of materials ensures that the cylindrical support only produces circumferential deformation under massive torque action. Regular deformation makes the influence of misalignment error on torque measurement precision reduced as much as possible. In the acquisition of signals, we adopted the measurement and control equipment of PXIE. The torque information is inputted into the FPGA programming module and then transmitted to the signal processing module for subsequent calculations.

\section{Analysis of the Additional Torque}

The measurement results of the torque transducers in the robot reducer detectors cannot be taken as the input and output torques of the robot reducer [21-24]. There is a distance between the torque transducers in the precision robot reducer detector and the reducer to be tested. The red parts when the TA is placed on the test location consist of a bearing, a spline sleeve, and a spline shaft, as is shown in Figure 3. The weights of components of the red parts are unequal, and misalignment errors result in uneven frictional force around them [25-27]. These factors bring about unbalanced stress distributions at the supports, which can further cause bending and torsional deformation of the shafts. So, additional torques are included in torque measurement results. The main factors causing additional torque in the precision robot reducer detector include the friction of the bearings and the misalignment error of the shafting. The following is an analysis of the two main parts of the additional torques using the torque transducer in the TMMISR and the input side of the tested reducer as an example.

3.1. Additional Torque Caused by Friction of Bearings. Regardless of the type of bearings, the friction torque is generated on the transmission shaft itself. The magnitude of friction torque is related to the turning inertia of the rotator, its rotational speed, and its material. Traditional friction torque compensation requires six parameters to be identified, and the calculation results are problematic. Since the stiffness of the shaft is related to the friction torque of the shaft, the maximum friction torque of the shaft can be obtained by calculating the maximum deformation angle of the shaft under the action of maximum torque. The polar inertia moment $K$ of the shaft is as follows:

$$
K=0.5 \pi\left(\left(\frac{D}{2}\right)^{4}-\left(\frac{d}{2}\right)^{4}\right)
$$




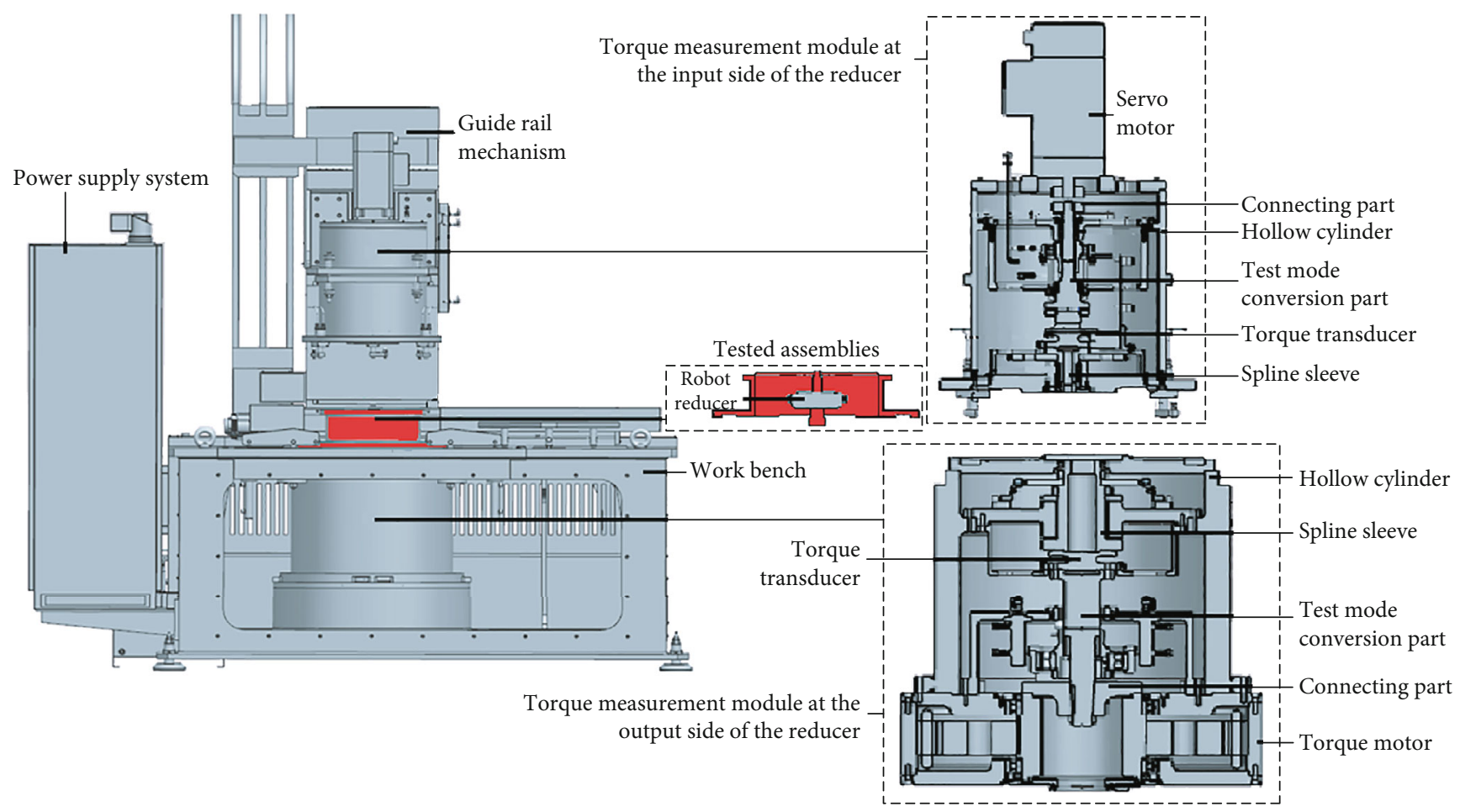

FIGURE 2: The vertical precision robot reducer detector.

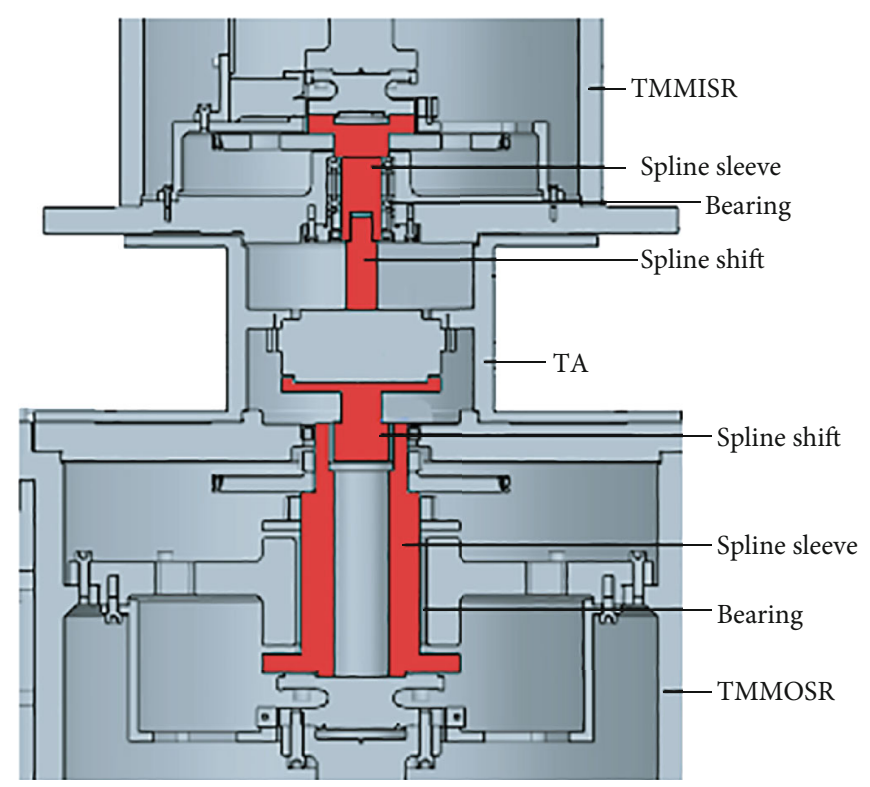

FIgURE 3: The parts leading to additional torques.

where $D$ is the outer diameter of the shaft and $d$ is the inner diameter of the shaft. For the solid shaft, the value of $d$ is zero. The maximum deformation angle of the shaft is calculated as follows: where $L$ is the length of the driveshaft, $T$ is the torque measured by the torque transducer in the instrument, and $G$ is the shear modulus of elasticity. The calculation of the maximum friction torque of the shaft is as follows:

$$
\beta=\frac{T L}{K G},
$$

$$
\Delta T_{1}=k_{o} \beta=\frac{32 k_{o} T L}{G \pi\left(D^{4}-d^{4}\right)},
$$


where $k_{o}$ is the conversion coefficient, which is linear to the radial offset of shaft $\Delta X$. That is,

$$
k_{o}=\mu \Delta X,
$$

where $\mu$ is the elastic coefficient. The radial offset of shaft $\Delta X$ is not only related to the coaxiality of shafting $\Delta r$ but also linearly related to the loaded radial force $F_{r}$. That is,

$$
\Delta X=\Delta r+\frac{F_{r}}{\varepsilon},
$$

where $\varepsilon$ is the strain coefficient. There is a linear relationship between the loaded radial force $F_{r}$ and the torque measured by the torque transducer in the instrument. That is,

$$
F_{r}=\frac{T}{r},
$$

where $r$ is the radius of the shaft. Thus, $k_{o}$ is linear to the applied torque; that is,

$$
k_{o}=\mu \Delta r+\frac{T}{r \varepsilon} .
$$

The additional torque caused by the friction of bearings is quadratic dependent on the torque measured by the torque transducer in the instrument:

$$
\Delta T_{1}=\frac{32(\mu \Delta r+T / a \varepsilon) T L}{G \pi\left(D^{4}-d^{4}\right)}=\frac{32 \mu \Delta r T L}{G \pi\left(D^{4}-d^{4}\right)}+\frac{32 T^{2} L}{a \varepsilon G \pi\left(D^{4}-d^{4}\right)} .
$$

3.2. Additional Torque Caused by Misalignment Error. Spline sleeve and spline shaft play a significant role in the system. Under an ideal working status, the torque transducer and the tested robot reducer should be oriented on the same central line. However, in actual production, there is no ideal situation where the torque transducer and the tested robot reducer are present on the same central line. The manufacturing errors of parts and the assembling errors lead to a deviation of the axle centerline. The radial misalignment error $\Delta X$ between the torque transducer and the tested robot reducer is shown in Figure 4.

In Figure $4, Z_{1}$ represents the axis of the torque transducer in the instrument and $Z_{2}$ represents the axis of the tested robot reducer. $\Delta X$ represents the offset of the $Z_{2}$ axis and the $Z_{1}$ axis in the radial direction. Here, the spline sleeve and spline shaft produce certain deformation. The force produced at the place where the torque transducer in the instrument can be expressed as follows:

$$
F=K \Delta X
$$

where $K$ is the elastic coefficient of the coupling and $\Delta X$ is the radial error. When there is a radial error $\Delta X$, the torque measured by the torque transducer is the composite moment of the bending moment and the torque. The torque transferred to the tested robot reducer is smaller than the torque mea- sured by the torque transducer. The additional torque caused by the radial error is as follows:

$$
\Delta T_{2}=F L=K L \Delta X,
$$

where $L$ is the length of the driveshaft and $\Delta X$ is not only related to the coaxial error of the shaft $\Delta r$ but also linearly related to the axial force $F_{r}$. That is,

$$
\Delta X=\Delta r+\frac{F_{r}}{\varepsilon},
$$

where $\varepsilon$ is the strain coefficient. There is a linear relationship between the loaded axial force $F_{r}$ and the torque measured by the torque transducer in the instrument. That is,

$$
F_{r}=\frac{T}{r},
$$

where $r$ is the radius of the shaft. Thus, the additional torque $\Delta T_{2}$ caused by the radial error is as follows:

$$
\Delta T_{2}=K L\left(\Delta r+\frac{T}{r} \varepsilon\right) .
$$

Misalignment errors consist of radial and angular displacement. The angular misalignment error $\Delta \theta$ between the torque transducer in the instrument and the tested robot reducer is shown in Figure 5.

In Figure $5, Z_{1}$ represents the axis of the torque transducer in the instrument and $Z_{2}$ represents the axis of the tested robot reducer. $\Delta \theta$ represents the angular misalignment error between the $Z_{2}$ axis and the $Z_{1}$ axis.

When there is an angular misalignment error $\Delta \theta$, the torque measured by the torque transducer in the instrument is the composite moment of the bending moment and the torque. The torque transferred to the tested robot reducer is smaller than the torque measured by the torque transducer. The additional torque caused by the angular misalignment error is as follows:

$$
\Delta T_{3}=T(\cos \Delta \theta-1)+\frac{T \cos \Delta \theta L}{r},
$$

where $L$ is the length of the driveshaft, $\Delta \theta$ is the angular misalignment error, $T$ is the torque measured by the torque transducer in the instrument, and $r$ is the radius of the shaft. By combining formulas (8), (13), and (14), the relationship between the additional torques $T^{\prime}$ and the torque measured by the torque transducer in the instrument $T$ can be obtained as follows:

$$
\begin{aligned}
T^{\prime}= & \Delta T_{1}+\Delta T_{2}+\Delta T_{3}=\frac{32 \mu \Delta r T L}{G \pi\left(D^{4}-d^{4}\right)}+\frac{T^{2}}{G \pi a \varepsilon\left(D^{4}-d^{4}\right)} \\
& +K L \Delta r+K L \frac{T}{a} \varepsilon+T(\cos \Delta \theta-1)+\frac{T \cos \Delta \theta L}{r} .
\end{aligned}
$$




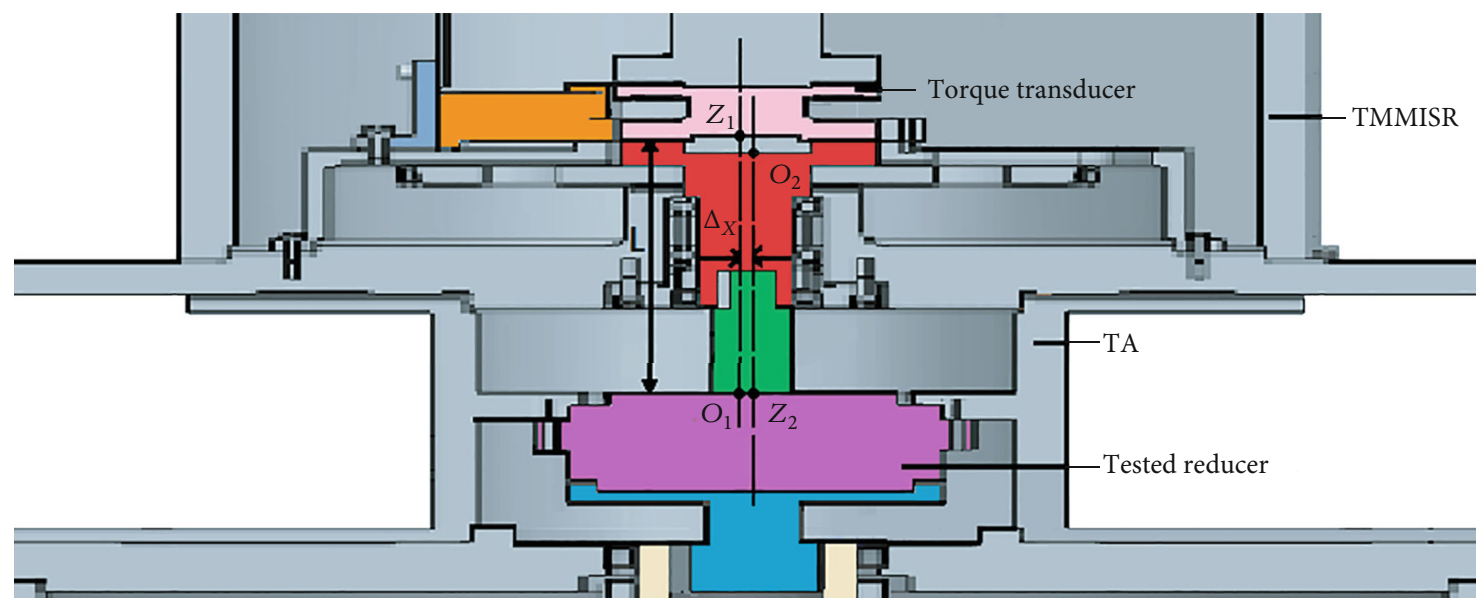

FIGURE 4: The radial misalignment error $\Delta X$.

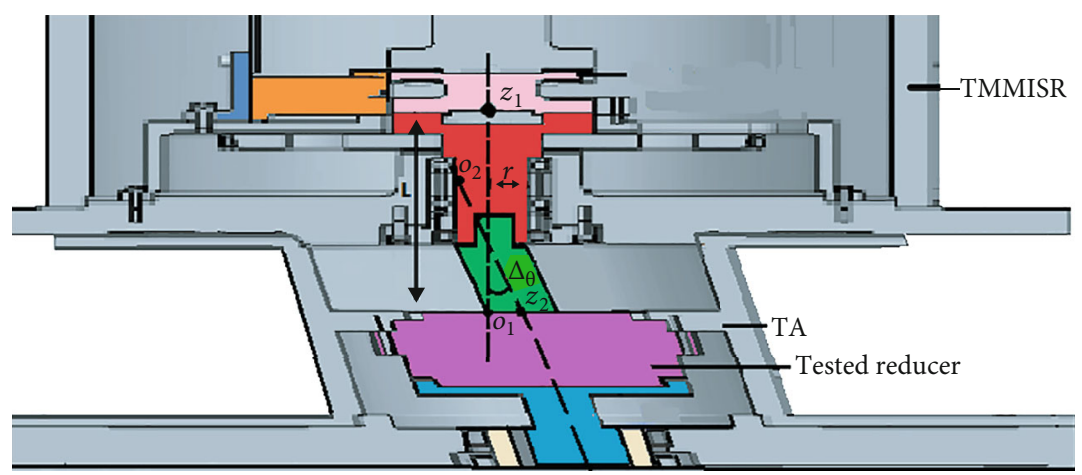

FIGURE 5: The angular misalignment error $\Delta \theta$.

We can conclude that the additional torques are quadratic dependent on the torque measured by the torque transducer in the instrument. If the relationship between the measurement results of the torque transducer and additional torques is obtained, the input and output torques of the reducer can be measured with high precision.

\section{Torque Calibration and Error Compensation}

As is analyzed in Section 3, additional torques are included in the measurement results of the torque transducers in the instrument. The system precision of the torque transducers cannot be taken as the measurement precision of the instrument. So, torque calibration and error compensation are applied to improve precision. This paper is an extension of the proceedings article [28]. Unlike that article, in which a method of calibrating the torque transducer is proposed, this paper mainly focuses on improving the accuracy of TMMISR and TMMOSR, since we have noticed the fact that the precision of the torque transducers cannot be considered the measurement precision of the torque-measurement systems.

4.1. Torque Calibration Method. It is required that the system output with known measurement accuracy is compared with the measurement results of the torque transducers to ensure accuracy in the measurement range $[29,30]$. In general, highprecision instruments or sensor systems are used as standards in a calibration process.

A torque calibrator is designed to deal with torque calibration, as is shown in Figure 6. The calibrator is certified by an authoritative metrological institution and enabled to output standard torque. It is placed on the position that the TA is located and reflects the real input and output torques of the reducer. Two high-precision torque output systems are in the calibrator, which calibrates torque transducers in TMMISR and TMMOSR, respectively.

When in calibration, the calibrator replaces the position of TA. In the motor output torque, the readings from the instrument and the torque calibrator are recorded simultaneously, and the output torque of TMMISR and TMMOSR is calibrated separately. The standard torque values of the two outputs are discrete, and 50 data are of average distribution in the range.

4.2. Error Compensation Model. A necessary process of highprecision measurement is to compensate for errors of the torque transducer. It requires to fit data over the whole range according to 50 groups of discrete standard torque values.

As analyzed in Section 3, there is a quadratic relationship between the measurement results of the torque transducer in 


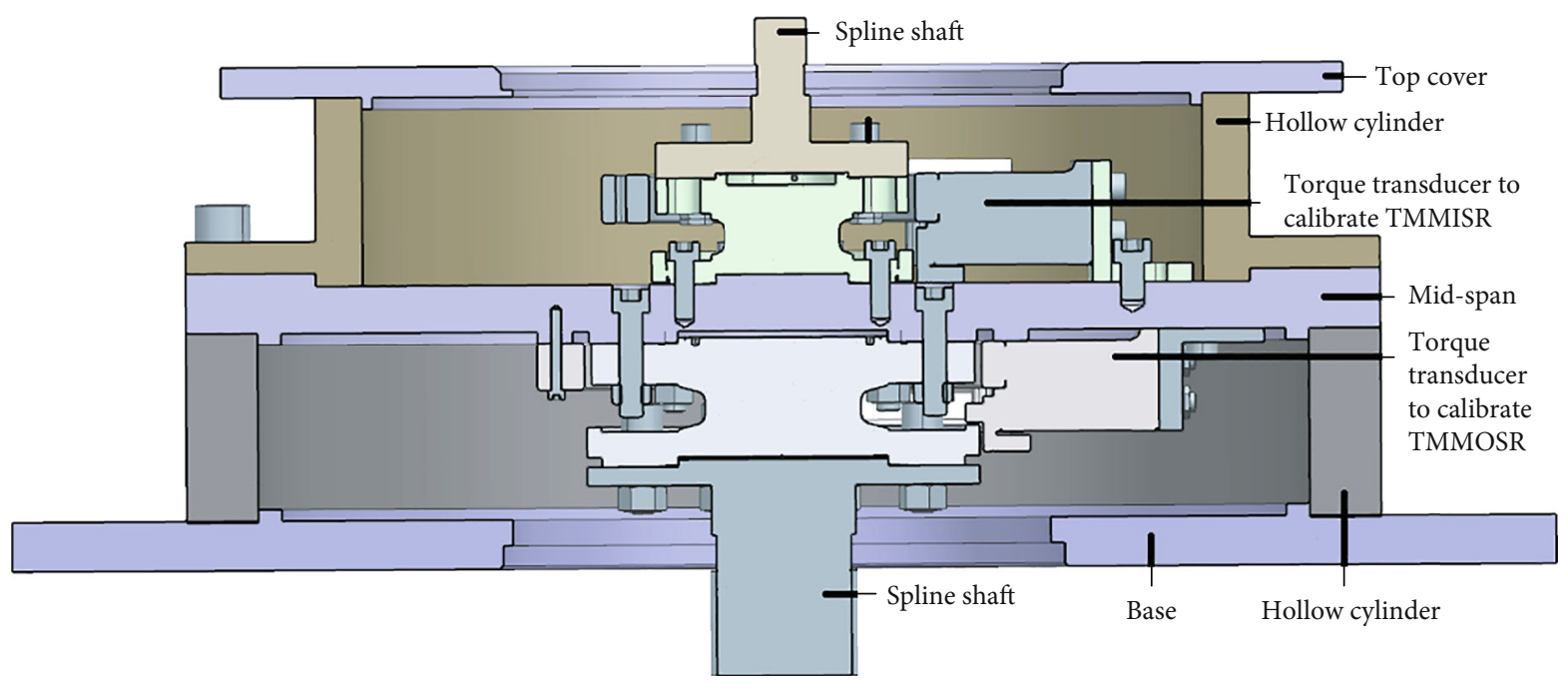

Figure 6: A design of the torque calibrator for the instrument.

the precision robot reducer detector and the additional torques. So, polynomial fitting can be used for compensation. But the accuracy of the polynomial fitting is limited by sampling points. Because there are only 50 groups of discrete standard torque values, the compensation effect is not ideal. By contrast, the BP neural network algorithm allows that error characteristics need not be focused on, so it is adopted in our error compensation process.

The three-layer feedforward neural network based on the BP algorithm can realize nonlinear mapping from input to output [31]. It can effectively simulate the relationship between the measurement results of the torque transducer in the instrument and the additional torques, which ensures a perfect fitting. As shown in Figure 7, the BP neural network algorithm used for torque error compensation is composed of multilayer mapping networks with unidirectional propagation. The networks include input, output, and hidden layers. The input layer contains one node denoted as $T_{i}$ that is the measurement results of the torque transducer in the instrument. The number of element nodes in the hidden layer is fifty, which is determined by experiments. The output layer contains one node denoted as $T_{I}^{\prime}$ that is the additional torques. The additional torques are calculated using the standard torque values minus the measurement results of the torque transducer in the instrument. The connection weight from $T_{i}$ to $G_{j}$ is $w_{i j}$, and that from $G_{j}$ to $T_{i}^{\prime}$ is $v_{j i}$. The transfer function of the hidden layer is the Tansig function, and that of the output layer is the Purlin function. The training function is the Traingdx function that can train a feedforward network with a fast BP algorithm.

In the experiment, the learning samples of measurement results of the torque transducer in the instrument and the corresponding additional torques are selected for training. The output of the hidden layer node is

$$
G_{j}=f\left(\sum_{j=1}^{50} w_{i j} T_{i}-b_{j}\right) .
$$

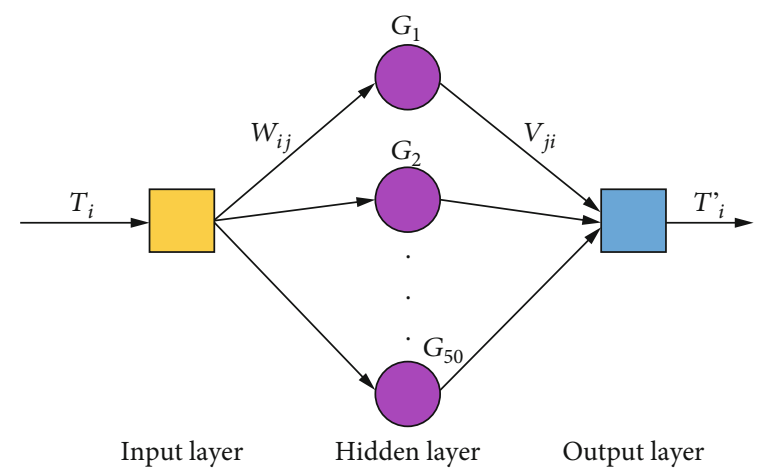

FIgURE 7: Torque error compensation model based on BP neural network.

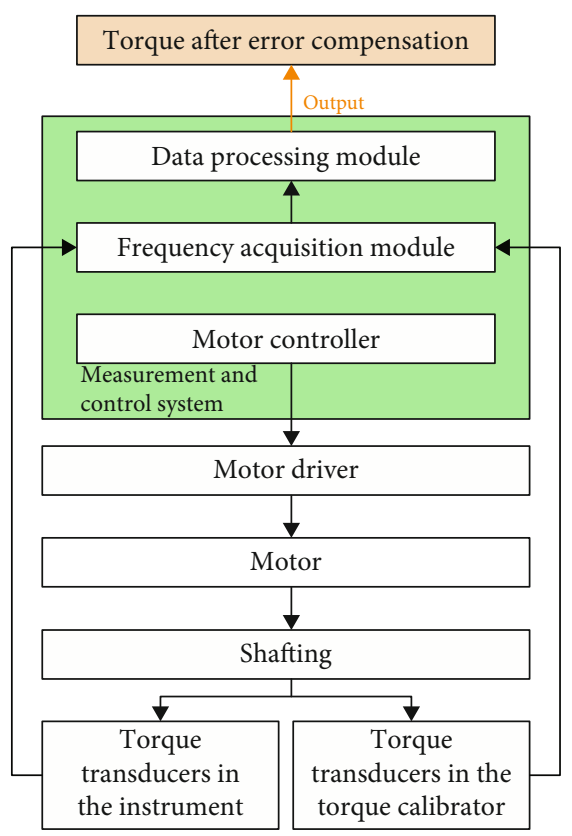

FIgURE 8: Process of automatic torque calibration. 


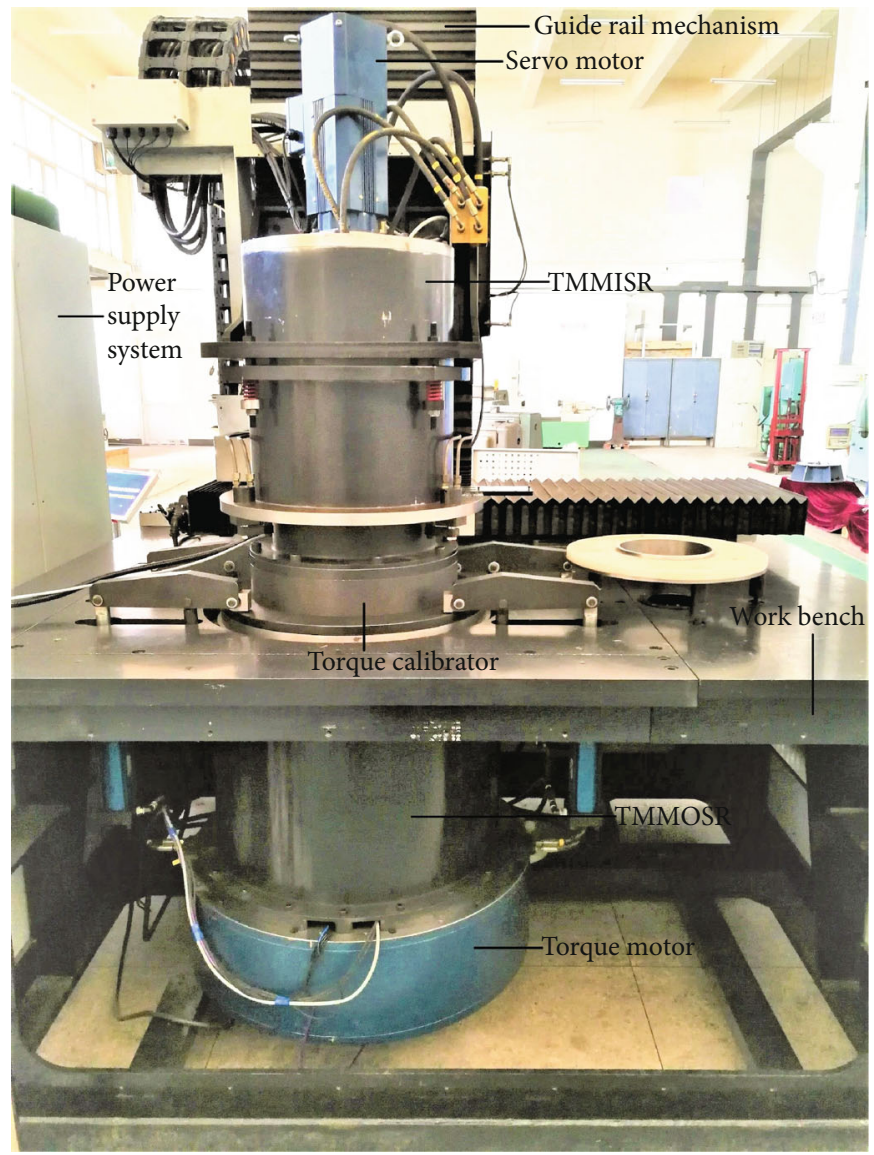

FIgURE 9: The experiment of calibration of the torque transducers in the instrument.

The output layer node model is

$$
T_{i}^{\prime}=f\left(\sum_{j=1}^{50} v_{j i} G_{i}-d_{j}\right) .
$$

In the above equations, $f$ is the action function and $b_{j}$ and $d_{j}$ are thresholds of the nerve units. The connection weights and thresholds are modified to calculate their influences on the total error from the last layer forward. The total error calculation function is

$$
e=\left(T_{i}^{\prime}-t_{i}\right)^{2}
$$

Here, $t_{i}$ is the calculated value of the output node. The appropriate network structure and parameters can be obtained by adjusting the connection weights and thresholds. The final training network with better fitting effects is used to compensate for torque errors.

\section{Experiment and Result}

5.1. Design of Automatic Calibration System. Torque calibration and error compensation model should be applied to the designed instrument with an automatic method.
Figure 8 shows the process of automatic torque calibration in our instrument.

The motor control module in the measurement and control system sends a command to the motor driver and then drives the motor to load a corresponding torque. So, the shafting transmits appointed torque, and the torque transducers in the instrument and the torque calibrator bear torque synchronously. The frequency signals output by the torque transducers in the instrument and the torque calibrator are sent to the data acquisition module during the loading process. The signals collected by torque transducers in the instrument are acquired and verified to be the original measured values. The signals collected by the torque calibrator are the standard value of input and output torques of the robot reducer and used as the reference values. Comparing reference values and original measured values in the synchronous acquisition, we can obtain errors in the calibration process. The data processing module then fits the compensation curve by using these obtained errors. This step is realized with the BP neural network. In the measurement process of the tested reducer, this compensation model is adopted to correct the errors of torque signals. Finally, torque data after error compensation are outputted and displayed, which are also taken as the final measurement values.

5.2. The Test and Verification of the Measuring Method. According to the designed scheme and the method described 


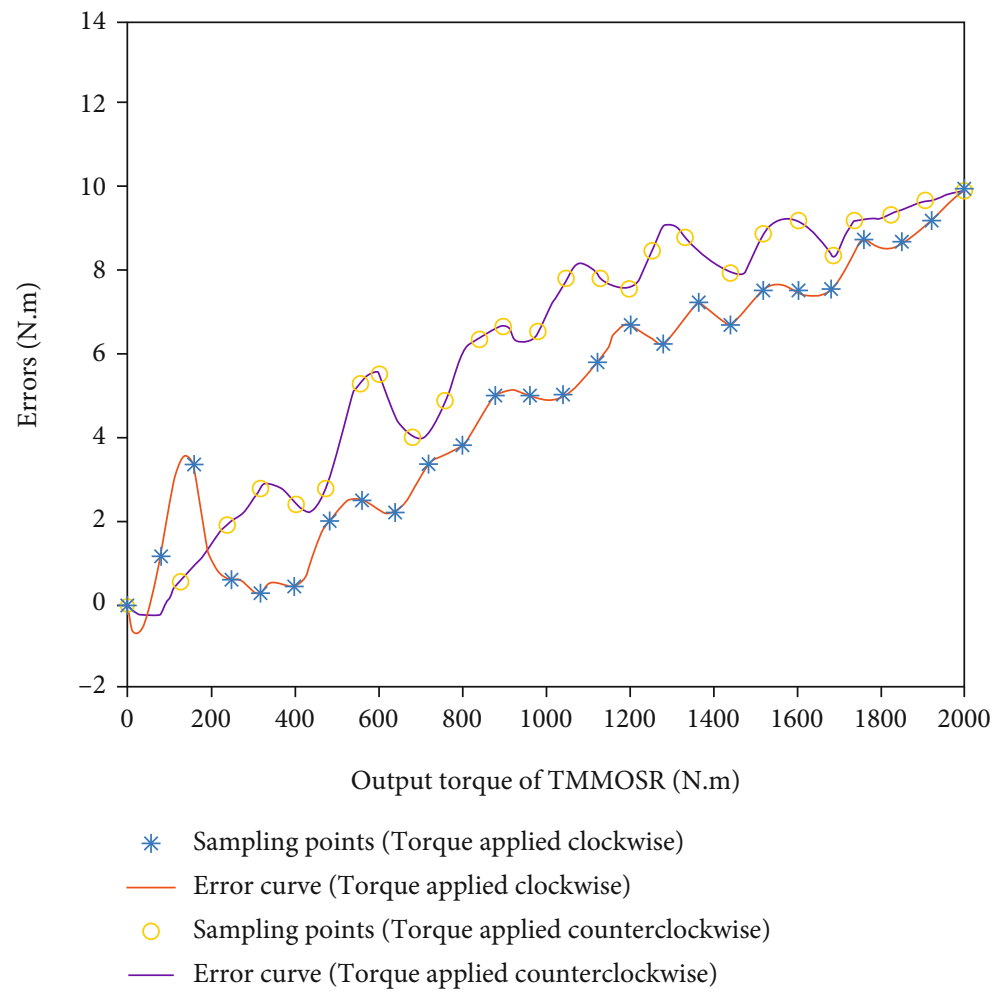

FIGURE 10: Error fitting curve of the output torque of TMMOSR.

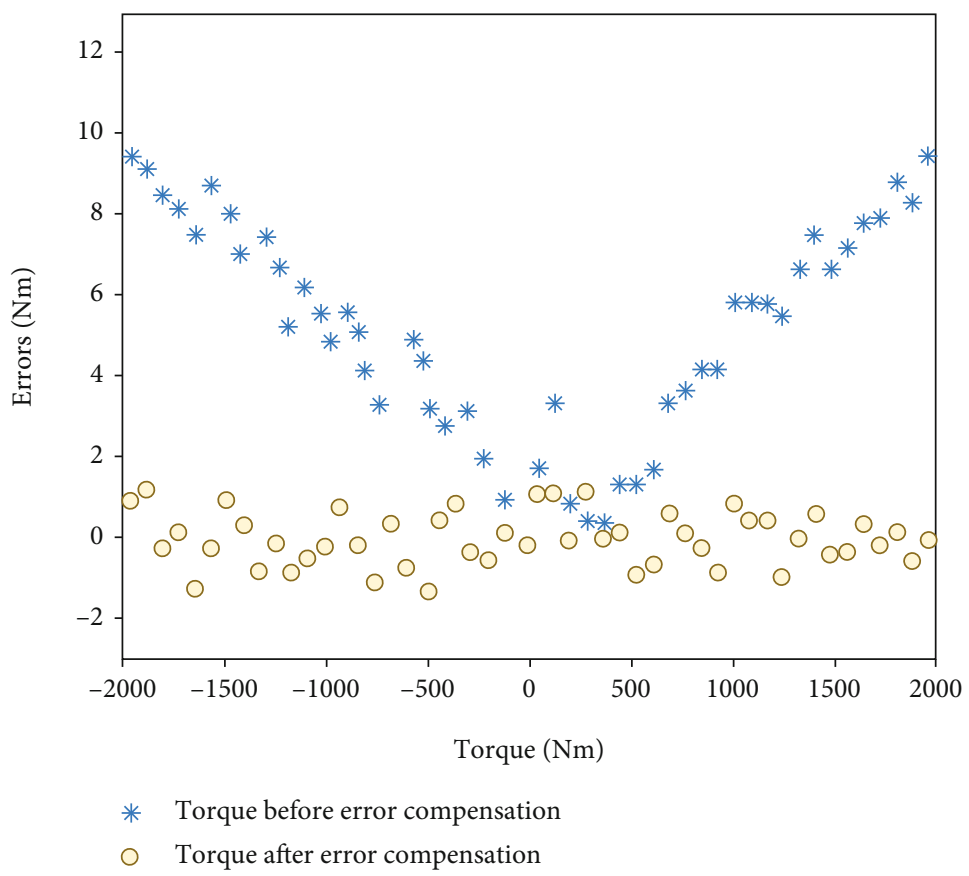

FIGURE 11: The errors of torque measurement systems before and after compensation.

in Sections 2-4, the torque calibration system was built according to the structural characteristics of the vertical instrument. As shown in Figure 9, in the experiment of calibration of the torque transducers in the instrument, the torque calibrator was placed at the test station, and we conducted the planned experiment steps of calibration according to the method of torque calibration in Section 4. The motors in the instrument applied torque step by step, clockwise or counterclockwise. We recorded the output torque and used the BP neural network to compensate for the required measured value in the torque range. The error curve is shown in Figure 10, which used the method in Section 4. 
The higher precision torque identified by the National Institute of Metrology (NIM) of China is used to verify the compensation effect of additional torques in the instrument and determine the torque output accuracy in TMMISR and TMMOSR. The torque measurement range of TMMISR is $\pm 50 \mathrm{Nm}$, and that of TMMOSR is $\pm 2000 \mathrm{Nm}$. The positive or negative sign represents the force direction that is clockwise or counterclockwise. 50 sampling points in each range are chosen for comparative analysis. A group of comparison results between the output torque of TMMOSR before and after error compensation with the standard torque is shown in Figure 11.

The test results show that the maximum error is $1.8 \mathrm{Nm}$, accounting for $0.09 \%$ of the whole measurement range. Through ten groups of verifications such as repeating experiments and choosing other sampling points, the torque measurement accuracy of the instrument can reach $0.1 \%$ in full scale.

\section{Conclusion}

In this paper, we proposed a torque-measurement system to measure the input and output torques of a high-precision reducer for industrial robots. Torque transducers were located in close proximity to the robot reducer to ensure the shortest measurement chain. The overall rigidity was found to be improved through structural optimisation. The symmetrical structure, with the cylindrical measurement modules and disk supports, simplified the deformation model and enabled easy error compensation. The characteristics of torque measurement errors were also analyzed by introducing their main causal factors and analyzing two main parts of the torque measurement errors. For the calibration and error compensation, a torque calibrator was designed to calibrate the torque transducers in the torque-measurement system. A BP neural network was used to compensate for torque measurement errors. Experimental results verifying the validity of the error compensation model showed that the precision of the torque-measurement system reached $0.1 \%$ in full scale. Moreover, most torque measurement errors were compensated. All the design and calibration methods ensured that the instrument has higher performance than other common testing instruments.

The limitation of this study is that the TMMISR and TMMOSR in this paper are calibrated under static conditions. However, the instrument is not only limited to static measurement but also excellent in dynamic measurement. And in the actual dynamic test, the error will vary with the speed, bearing lubrication, and temperature. Therefore, in order to improve the dynamic accuracy of torque measurement, more experiments and model studies will be carried out in the next step to find out the causes of errors and further improve the measurement accuracy of the instrument.

\section{Data Availability}

The data used to support the findings of this study are available from the corresponding author upon request.

\section{Conflicts of Interest}

The authors declare that they have no conflicts of interest.

\section{References}

[1] E. Bahadir, "Prediction of prospective mathematics teachers' academic success in entering graduate education by using back-propagation neural network," Journal of Education and Training Studies, vol. 4, no. 5, pp. 113-122, 2016.

[2] X. Chu, H. Xu, X. Wu, J. Tao, and G. Shao, "The method of selective assembly for the RV reducer based on genetic algorithm," Proceedings of the Institution of Mechanical Engineers, Part C: Journal of Mechanical Engineering Science, vol. 232, no. 6, pp. 921-929, 2018.

[3] F. Deng, K. Li, X. Hu, H. Jiang, and F. Huang, "Life calculation of angular contact ball bearings for industrial robot RV reducer," Industrial Lubrication and Tribology, vol. 71, no. 6, pp. 826-831, 2019.

[4] W. Fu, C. Ai, C. Tang, Y. Yang, G. Li, and H. Zhao, "RV reducer dynamic performance test bed design in closed power flow," in Proc. of the 4th International Conference on Sensors, Mechatronics and Automation, pp. 666-669, Zhuhai, China, 2016.

[5] T. Li, J. Zhou, X. Deng et al., "A manufacturing error measurement methodology for a rotary vector reducer cycloidal gear based on a gear measuring center," Measurement Science and Technology, vol. 29, no. 7, article 075006, 2018.

[6] M. Iwasaki and H. Nakamura, "Vibration suppression for angular transmission errors in harmonic drive gearings and application to industrial robots," IFAC Proceedings Volumes, vol. 47, no. 3, pp. 6831-6836, 2014.

[7] P. Peng and J. G. Wang, "NOSCNN: a robust method for fault diagnosis of RV reducer," Measurement, vol. 138, pp. 652-658, 2019.

[8] A. D. Pham and H. J. Ahn, "High precision reducers for industrial robots driving 4th industrial revolution: state of arts, analysis, design, performance evaluation and perspective," International Journal of Precision Engineering and Manufacturing-Green Technology, vol. 5, no. 4, pp. 519533, 2018.

[9] H. Xu, Z. Shi, B. Yu, and H. Wang, "Dynamic measurement of the lost motion of precision reducers in robots and the determination of optimal measurement speed," Journal of Advanced Mechanical Design, Systems, and Manufacturing, vol. 13, no. 3, p. 15, 2019.

[10] X. Sun, L. Han, K. Ma, L. Li, and J. Wang, "Lost motion analysis of CBR reducer," Mechanism and Machine Theory, vol. 120, pp. 89-106, 2018.

[11] T. L. Tran, A. D. Pham, and H. J. Ahn, "Lost motion analysis of one stage cycloid reducer considering tolerances," International Journal of Precision Engineering and Manufacturing, vol. 17, no. 8, pp. 1009-1016, 2016.

[12] T. D. Tuttle and W. P. Seering, "A nonlinear model of a harmonic drive gear transmission," IEEE Transactions on Robotics and Automation, vol. 12, no. 3, pp. 368-374, 1996.

[13] J. G. Kim, Y. J. Park, G. H. Lee, S. D. Lee, and J. Y. Oh, “Experimental study on the carrier pinhole position error affecting dynamic load sharing of planetary gearboxes," International Journal of Precision Engineering and Manufacturing, vol. 19, no. 6, pp. 881-887, 2018. 
[14] J. G. Kim, Y. J. Park, G. H. Lee, and J. H. Kim, “An experimental study on the effect of carrier pinhole position errors on planet gear load sharing," International Journal of Precision Engineering and Manufacturing, vol. 17, no. 10, pp. 13051312, 2016.

[15] M. Ruderman and M. Iwasaki, "Sensorless torsion control of elastic-joint robots with hysteresis and friction," IEEE Transactions on Industrial Electronics, vol. 63, no. 3, pp. 18891899, 2016.

[16] Y. Cai, L. Yao, J. Zhang, Z. Xie, and J. Hong, "Feasibility analysis of using a two-stage nutation drive as joint reducer for industrial robots," Journal of Mechanical Science and Technology, vol. 33, no. 4, pp. 1799-1807, 2019.

[17] F. Concli, "Thermal and efficiency characterization of a lowbacklash planetary gearbox: an integrated numericalanalytical prediction model and its experimental validation," Proceedings of the Institution of Mechanical Engineers, Part J: Journal of Engineering Tribology, vol. 230, no. 8, pp. 9961005, 2016.

[18] Y. G. Sun, X. F. Zhao, F. Jiang, L. Zhao, D. Liu, and G. B. Yu, "Backlash analysis of RV reducer based on error factor sensitivity and Monte-Carlo simulation," International Journal of Hybrid Information Technology, vol. 7, no. 2, pp. 283-292, 2014.

[19] R. B. Wang, H. Xu, B. Li, and Y. Feng, "Research on method of determining hidden layer nodes in BP neural network," Computers and Technology Development, vol. 28, no. 4, pp. 31-35, 2018.

[20] R. Wang, F. Gao, M. Lu, and T. Liu, "Meshing efficiency analysis of modified cycloidal gear used in the RV reducer," Tribology Transactions, vol. 62, no. 3, pp. 337-349, 2019.

[21] J. Xue, Z. Qiu, L. Fang, Y. Lu, and W. Hu, "Angular measurement of high precision reducer for industrial robot," IEEE Transactions on Instrumentation and Measurement, vol. 70, pp. 1-10, 2021.

[22] H. Xu and X. Liu, "Analysis for assembly dimension chain of RV reducer," Applied Mechanics and Materials, vol. 635-637, pp. 1826-1829, 2014.

[23] Y. H. Yang, C. Chen, and S. Y. Wang, "Response sensitivity to design parameters of RV reducer," Chinese Journal of Mechanical Engineering, vol. 31, no. 1, p. 49, 2018.

[24] Z. Zhang, J. Wang, G. Zhou, and X. Pei, “Analysis of mixed lubrication of RV reducer turning arm roller bearing," Industrial Lubrication and Tribology, vol. 70, no. 1, pp. 161-171, 2018.

[25] S. W. Hejny, Design of a harmonic drive test apparatus for data acquisition and control, [M.S thesis], Rice University, 1997.

[26] F. H. Ghorbel, P. S. Gandhi, and F. Alpeter, "On the kinematic error in harmonic drive gears," Journal of Mechanical Design, vol. 123, no. 1, pp. 90-97, 2001.

[27] O. O. Ogidi, P. S. Barendse, and M. A. Khan, "Measuring fault indicators in electric machines-learning experience," IEEE Instrumentation \& Measurement Magazine, vol. 21, no. 5, pp. 52-53, 2018.

[28] Y. Zhen and Y. Wancheng, "A static calibration system of torque sensor based on BP neural network," Chinese Journal of Sensors and Actuators, vol. 33, no. 2, pp. 238-244, 2020.

[29] N. Saenkhum and T. Sanponpute, "The optimization of continuous torque calibration procedure," Measurement, vol. 107, pp. 172-178, 2017.
[30] Y. K. Park, M. S. Kim, J. T. Lee, H. Y. Lee, and D. I. Kang, “Torque traceability examination of calibration laboratories in Korea," Measurement, vol. 42, no. 10, pp. 1443-1447, 2009.

[31] Y. Wang, B. Niu, H. Wang, N. Alotaibi, and E. Abozinadah, "Neural network-based adaptive tracking control for switched nonlinear systems with prescribed performance: an average dwell time switching approach," Neurocomputing, vol. 435, no. 6, pp. 295-306, 2021. 